\title{
Optimization Design of Mathematical Modeling for Leakage Noise Reduction of Bionic Fan Blade
}

\author{
Lizong Cao ${ }^{1, a}$, Yu Zhang ${ }^{2, b}$, Nannan Zhao ${ }^{3, c}$ and Xiaoxu Chen ${ }^{4}$ \\ 1,2,3,4 Jilin Agricultural University, Changchun, Jilin,130118, China \\ aleezs643@sina.com, 'blzs036@163.com, ${ }^{\mathrm{C}} 499360221 @ q q . c o m$
}

Keywords: bionic, noise reduction, working parameters, variance analysis

\begin{abstract}
We used the characteristics of the birds' wings, the blades of the fan are improved, and several factors that influence the noise of the bionic leaves are experimentally analyzed. Finally, the striped width of the leaves, the stripes of the leaves and the thickness of the leaves are taken as factors A, B, and C. The parameters of the bionic fan are measured and recorded. The data are analyzed by the difference and variance analysis. The relevant noise reduction data are obtained and the mathematical model is established by using the data to facilitate the noise reduction effect of the bionic fan.
\end{abstract}

\section{Introduction}

Fan blade noise sources include aerodynamic noise and mechanical noise, where we mainly the discussion of aerodynamic noise. Pneumatic noise is the noise generated by the interaction of gas during the flow and the fan blades, ducts and rectifiers. We can improve the noise by improving the structure of the fan, the shape of the blade, the material of the blade and the duct. And the advantages of bionic leaves here have been fully reflected, there is a better noise reduction effect.

We use the characteristics of the bird wing, combined with the fan, the axial fan blade bionic deformation, respectively, in the blade surface or edge set the jagged form, stripes and concave shape, in order to test the modified fan blades Whether the effect of reducing the noise, we combined with the original fan blade with the experimental comparison, the results show that the bionic transformation of the leaves in the noise reduction is indeed more superior than the original leaves.In order to further explore the factors affecting the noise reduction effect of bionic leaves, we have made orthogonal experiments on striped bionic leaves.The spacing of the stripes and the thickness of the blades are set to three influencing factors A, B and C respectively. Each factor has two levels.Through the comparison of experimental results[1].

\section{Extreme analysis of experimental conditions and experimental results}

Experimental method:The purpose of the experiment is to investigate the effect of different horizontal parameters on the noise generation during the operation of the blade. Through the comparison of the experimental results, the main factors affecting the noise reduction effect are determined, and the effect of the noise reduction is different And the mathematical model is used to study and analyze the noise reduction of bionic leaves. In order to reduce the experimental measurement error and the impact of the outside world on the measurement results, each of the different levels of the parameters of the bionic leaf experiment to do three times, the statistical results, the average value of the analysis[2]. Specific experimental parameters in Table 1.

Table 1 Bionic leaf noise reduction effect of experimental factors level table

\begin{tabular}{|l|l|l|}
\hline \multirow{2}{*}{ Factor } & \multicolumn{2}{|c|}{ Level } \\
\cline { 2 - 3 } & \multicolumn{2}{|c|}{1} \\
\hline Blade stripes width A (mm) & 0.5 & 1.0 \\
\hline Leaf stripes spacing B (mm) & 0.5 & 1.0 \\
\hline Blade thickness C (mm) & 0.2 & 0.4 \\
\hline
\end{tabular}

For the different levels of the bionic leaves were orthogonal arrangement, see Table 2 
Table 2

\begin{tabular}{|c|c|c|c|c|c|c|}
\hline \multirow{2}{*}{$\begin{array}{c}\text { Serial } \\
\text { number }\end{array}$} & \multicolumn{4}{|c|}{ Factor } & \multirow{2}{*}{$\begin{array}{c}\text { The average of } \\
\text { the noise of the } \\
\text { bionic leaves } \\
\text { M }\end{array}$} & \multirow[b]{2}{*}{$M^{\prime}=M-77.4$} \\
\hline & $\mathrm{A}(\mathrm{x})$ & $\mathrm{B}(\mathrm{x})$ & & & & \\
\hline 1 & A1（0.5） & B1（0.5） & 1 & C1 (0.2) & 77.6 & 0.2 \\
\hline 2 & A1 & B2 (1.0) & 2 & C2 $(0.4)$ & 77.1 & -0.3 \\
\hline 3 & A2 (1.0) & $\mathrm{B} 1$ & 2 & $\mathrm{C} 2$ & 76.8 & -0.6 \\
\hline 4 & A2 & B2 & 1 & C1 & 77.9 & 0.5 \\
\hline$\sum M_{1 j}^{\prime}$ & -0.1 & -0.4 & 0.7 & 0.7 & \multirow{8}{*}{$T=\sum_{i=1}^{2} \sum_{j=1}^{2}(M$} & \\
\hline$\sum M_{2 j}^{\prime}$ & -0.1 & 0.2 & -0.9 & -0.9 & & \\
\hline $\bar{M}_{1 \mathrm{j}}$ & -0.05 & -0.2 & 0.35 & 0.35 & & \\
\hline $\bar{M}_{2 \mathrm{j}}^{\prime}$ & -0.05 & 0.1 & -0.45 & -0.45 & & \\
\hline$R_{\mathrm{j}}$ & 0 & 0.3 & 0.8 & 0.8 & & \\
\hline $\begin{array}{c}\text { Excellent } \\
\text { level }\end{array}$ & C2 & B1 & & A1/A2 & & \\
\hline $\begin{array}{c}\text { Primary and } \\
\text { secondary } \\
\text { factors }\end{array}$ & C & B & & A & & \\
\hline $\begin{array}{c}\text { Excellent } \\
\text { combination }\end{array}$ & $\mathrm{C} 2$ & B1 & & A1/A2 & & \\
\hline
\end{tabular}

\section{Analysis of variance of the effect of noise reduction in bionic leaves}

Test data processing, we use a very poor method can be very intuitive to discharge the primary and secondary factors, but there is a drawback is that data accuracy can not be drawn. So, here we use the variance method to get the analytical accuracy to overcome the shortcomings of the poor analysis.

From the above analysis of variance, we can see that the sum of squares of each factor can be calculated by the following formula[3].

$$
S_{\mathrm{j}}^{2}=\frac{1}{2}\left[\left(\sum M_{1 j}^{\prime}\right)^{2}+\left(\sum M_{2 j}^{\prime}\right)^{2}\right]-\frac{T^{2}}{4} \quad \mathrm{j}=1,2,3,4
$$

$S_{A}=S_{1}, S_{B}=S_{2}, S_{E}=S_{3}, S_{D}=S_{4}$

The sum of the squares of the total deviation is

$$
S_{T}^{2}=\sum_{i=1}^{2} \sum_{j=1}^{2} M_{i j}^{\prime 2}-\frac{T^{2}}{4}
$$

In the process of variance analysis, we can use the data corresponding to the third column (blank column) in Table 2 to estimate the experimental error. Through the analysis of variance, we can conclude that the effect of factor $\mathrm{C}$ on the noise reduction of bionic leaves is significant, factor $\mathrm{B}$ is significant, and factor A is not significant. From this we can see that the main factors that affect the noise reduction of the bionic fan blades are the blade thickness, followed by the blade fringe spacing, and the blade strip width has little or no effect on the noise reduction effect of the bionic fan blades. 


\section{Function of bionic leaf noise reduction experiment}

In the above experiment, we can see that the noise value $\mathrm{M}$ of the bionic leaves has an interdependent relationship with the blade thickness $\mathrm{x}$ and the blade fringe pitch $\mathrm{y}$, that is, they have a functional relationship. By the previous analysis of variance, we consider that there is no interaction between the factor $\mathrm{C}$ and the factor $\mathrm{B}$, and the influence of the thickness $\mathrm{x}$ and the blade fringes $y$ on the noise of the bionic leaves is independent of each other. Therefore, we can establish the relationship between leaf thickness and bionic leaf noise and leaf fringe spacing and bionic leaf noise, respectively[4].

(1) the function of bionic leaf noise and leaf thickness

From Table 2 we can see,when the blade thickness is $x_{1}=0.2, x_{2}=0.4$,corresponding to the average noise of the leaves were $M_{1}=77.75, M_{2}=76.95$, we can see that within a certain range, with the increase in blade thickness, the noise value of the bionic leaves is decreasing.Therefore, we can assume that there is a linear function relationship between the noise value $\mathrm{M}$ and fan blade thickness $\mathrm{x}$ of the bionic blade: $M=a x+b$.Using the data obtained above and the least squares method,we can estimate $a=-4, b=78.55$,thus the linear function between the bionic leaf noise $\mathrm{M}$ and the blade thickness $\mathrm{x}$ is $M=-4 x+78.55$. This can be used to study the relationship between blade thickness and bionic leaf noise values.

(2) The relationship between the noise of the bionic leaf and the spacing of the leaf stripes.

Similarly, we can also see in the table 2, when the blade fringe spacing is $y_{1}=0.5, y_{2}=1.0$,corresponding to the average noise of the leaves were $M_{1}=77.2, M_{2}=77.5$,we can see that in a certain range, with the increase in the pitch of the blade, the noise value of the bionic leaves increases.

Therefore, we can assume that there is a linear function relationship between the noise value $\mathrm{M}$ and leaf fringe spacing $y$ of the bionic leaf: $M=a y+b$.Using the data obtained above, Using the least squares method, we can estimate $a=0.6, b=76.9$, so the linear function between bionic leaf noise $\mathrm{M}$ and leaf stripe spacing is $M=0.6 y+76.9$.This can be used to study the relationship between leaf fringe spacing and bionic leaf noise values.

\section{Conclusion}

Through the above experimental data comparison we can see the impact of bionic fan blade noise value is the main factor in the thickness of the blade, we can increase the thickness of the blade as much as possible to reduce the blade noise value. The blade fringe spacing is a secondary factor affecting the blade noise. The noise value of the blade increases gradually with the increase of the fringe spacing. Therefore, it is necessary to reduce the blade fringe spacing as much as possible to achieve the purpose of noise reduction. While the leaf stripes on the leaves of the noise is relatively small or even no effect. We can properly adjust the blade stripes width, is conducive to other factors of improvement.

\section{Acknowledgement}

Jilin Aggricultural University National College Students Innovation and Entrepreneurship Training Program Project.

Jilin Agricultural University Undergraduate Science and Technology Innovation Fund Project.

\section{References}

[1] Luquan ren.Experimental optimization design and analysis[M].Beijing:Higher Education Press, 2003.

[2] Zenghui Wang.Effect of Working Parameters on Power Consumption of Universal Blade in 
Rotary Cropping[J].Journal of Jilin University (Engineering Science Edition), 2013, 42: 1-4.

[3] Zenghui Wang.Probability Theory and Mathematical Statistics[M].Beijing:Higher Education Press,2011.

[4] D.S.Miklosovic, M.M.Murray, L.E.Howle, F.E.Fish. Leading-edge tubercles delay stall on humpback whale(Megaptera novaeangliae)flippers. Physics of Fluids,2004 16(5):39-42. 